\title{
Effects of Aerobic Exercise on Lipid Profiles and High Molecular Weight Adiponectin in Japanese Workers
}

\author{
Wei Guo ${ }^{1}$, Hiroaki Kawano ${ }^{2}$, Lianhua Piao ${ }^{1}$, Nana Itoh ${ }^{3}$, Koichi Node $^{2}$ and Takeshi Sato ${ }^{4}$
}

\begin{abstract}
Background The metabolic syndrome is characterized by the accumulation of several metabolic risk factors. It is important to improve physical activity and dietary habits to reduce the risk of cardiovascular disease in humans.

Methods The study participants participated in a weekly aerobic exercise program that included a session composed of a brief meeting, warm-up exercises, and primary exercises (low and high impact, stretch, muscle training, and cooling down). To evaluate the effect of this intervention we measured body fat composition, holding power, and quality of life assessment. Blood tests were also carried out before and every 3 months during the study.

Results Of the 37 participants enrolled in the exercise group, 31 (83.8\%) completed the 12-week program. The control group consisted of 42 subjects, $36(85.7 \%)$ of whom were available for follow-up at the end of the 12-week study period. In the exercise group, weight, body fat percentage, waist circumference, the World Health Organization quality of life 26 (WHO-QOL 26) score, triglyceride, total cholesterol, high density lipoprotein cholesterol and low density lipoprotein cholesterol had improved significantly at the end of three months. The high molecular weight adiponectin concentration of the participants in the exercise group increased during the 9-month period of the study, although this change did not reach statistical significance compared with pre-exercise.

Conclusion Aerobic exercise led to an improvement in body composition and lipid profiles. High molecular weight adiponectin concentrations tended to improve compared with pre-aerobic exercise levels.
\end{abstract}

Key words: aerobic exercise, high molecular weight (HMW) adiponectin

(Intern Med 50: 389-395, 2011)

(DOI: 10.2169/internalmedicine.50.4380)

\section{Introduction}

The metabolic syndrome is characterized by the accumulation of several metabolic risk factors, such as abdominal obesity, dyslipidemia, hypertension, glucose intolerance, and prothrombotic and proinflammatory states (1-5). It is well known that patients with the metabolic syndrome have an increased risk of cardiovascular disease (6). Recently, the prevalence of patients with the metabolic syndrome has increased in Japanese workers. According to the International Diabetes Federation (IDF) and the National Cholesterol Education Program (NCEP) III criteria, the prevalence of the metabolic syndrome in Japanese workers is reported to range from $25.8 \%$ to $33.0 \%$ (7).

It is important to improve physical activity and dietary habits to reduce the risk of cardiovascular disease in humans. Several earlier studies have shown that the beneficial effects of aerobic exercise on blood pressure, cholesterol levels, and insulin sensitivity occur regardless of whether weight loss is achieved or not $(8,9)$. In fact, the Pawtucket Heart Study group reported there is a close relationship between physical activity and the levels of high density lipoprotein (HDL) cholesterol (10). A study in 3,000 adult Japanese men also showed that the frequency of physical activity correlated positively with HDL cholesterol levels (11).

${ }^{1}$ Doctoral Program, Saga University, Japan, ${ }^{2}$ Department of Cardiovascular and Renal Medicine, Saga University, Japan, ${ }^{3}$ Master's Program, Saga University, Japan and ${ }^{4}$ Health Care Center, Faculty of Medicine, Saga University, Japan

Received for publication August 17, 2010; Accepted for publication November 16, 2010

Correspondence to Dr. Takeshi Sato, satot@cc.saga-u.ac.jp 
The aims of the present study in Japanese workers were to assess the effects of long-term aerobic exercise on lipid profiles, glucose levels, and plasma high molecular weight (HMW) adiponectin, an anti-atherosclerotic adipokine.

\section{Materials and Methods}

\section{Participants}

Participants were enrolled in the study using newspaper and radio advertisements, and pamphlets. A total of 79 subjects aged 28-76 years (21 men and 58 women) were enrolled in the intervention group.

According to the Japanese diagnostic criteria, the metabolic syndrome is defined as a waist circumference of at least $85 \mathrm{~cm}$ in men, or $90 \mathrm{~cm}$ in women, plus at least two of the following characteristics: 1) triglycerides $\geq 1.69$ $\mathrm{mmol} / \mathrm{L}(150 \mathrm{mg} / \mathrm{dL})$ or HDL cholesterol $<1.03 \mathrm{mmol} / \mathrm{L}$ (40 $\mathrm{mg} / \mathrm{dL}$ ), 2) systolic blood pressure $\geq 130 \mathrm{mmHg}$ or diastolic blood pressure $\geq 85 \mathrm{mmHg}$, and 3) fasting plasma glucose $\geq 6.11 \mathrm{mmol} / \mathrm{L}$ (110 mg/dL) (12). The exclusion criteria in this study were a history of cardiovascular diseases, other serious illnesses, and type 1 diabetes mellitus. After the participants were asked about their history of physical and psychiatric illnesses, a physical examination was carried out on each participant.

The consent forms explained the purpose of this study, confidentiality of data, the possibility of declining to participate, and the contact numbers of the researchers. Documents containing explanations and precautions, and consent forms were distributed to the participants to obtain their written permission before the survey. They obtained and approval the written informed consent from 79 subjects in total. The study protocol was submitted to and approved by the Institutional Review Board (IRB) of the Faculty of Medicine at Saga University.

\section{Intervention}

The exercise program was undertaken 1 day each week for 3 months or more up to a maximum of 18 months. The three-month course therefore consisted of a total of 12 sessions. Each session was of 1.5 hours duration and consisted of a brief meeting (10 minutes), warm-up exercises ( 5 minutes), primary exercises (30-35 minutes), stretch exercises (10 minutes), muscle training (25 minutes), and cooling down (5 minutes). Participants were assigned training ranges using a pre-established heart rate equivalent to $70 \%$ to $85 \%$ of their maximum heart rate (13).

\section{Physical assessment}

Physical assessment including weight, body mass index (BMI), body fat percentage (\% body fat) was performed for each participant before the aerobic exercise program and 3 months later using the TANITA TBF-305 (Tanita, Tokyo, Japan) body fat/composition monitor. Waist circumference was measured at the navel with the subject in the erect position after relaxed breathing. Duplicate measurements of holding power of the left and right hand were recorded using a grip dynamometer, and the average of the values recorded. During these measurements, the subjects were requested not to move the dynamometer.

\section{QOL assessment}

We evaluated the participants' quality of life using the World Health Organization Quality of Life scale (WHOQOL) (14). The 26-item short form of the WHO-QOL is the brief version of the WHO-QOL 100, which was developed to assess subjects around the world, regardless of culture or local customs. It consists of 26 items classified into the following five domains: physical domain, psychological domain, social relationships, environmental domain, and general QOL. We evaluated the QOL assessment before the course and after three months of aerobic exercise.

\section{Blood tests}

The levels of triglyceride, total cholesterol, HDL cholesterol, low density lipoprotein (LDL) cholesterol, and fasting plasma glucose were measured during the study. Plasma HMW adiponectin level was measured using a sandwich ELISA kit (Fujirebio, Tokyo, Japan) that incorporated a monoclonal antibody to human HMW adiponectin, IH7 (15). A working standard of HMW adiponectin was prepared using human HMW adiponectin purified by passage through a Geratin-Cellulofine affinity column (Seikagaku Industrial Co., Tokyo Japan). The sensitivity and upper limit of the working range of HMW adiponectin levels were 0.18 to $22.05 \mu \mathrm{g} / \mathrm{mL}$. The intra- and inter-assay variances were 2.4 to $3.0 \%$ and 4.2 to $5.1 \%$, respectively.

\section{Statistical analysis}

The data were expressed as the mean \pm standard error (SE). The Chi-square test and Mann-Whitney $U$ test were used to compare the characteristics of the two groups. The Wilcoxon signed-rank test was used to compare the values before the program and after three months of aerobic exercise. A two-way repeated measures analysis of variance (ANOVA) (aerobic exercise duration $\times$ gender) was used to compare the values at 3, 6, 9, 12 and $>12$ months between the group performing exercises and pre-exercise. Pearson's correlation analysis and multiple regression analysis to examine the relationship between HMW adiponectin and the other factors and the Mann-Whitney $U$ test was used to compare HMW adiponectin concentrations at 3,6 and 9 months. The Statistical Package for the Social Sciences (SPSS, SPSS Japan Inc., Tokyo, Japan) software version 17 was used for the statistical analyses, with statistical significance being set at $\mathrm{p}$ values $<0.05$. 


\section{Results}

\section{Subjects}

Thirty-seven subjects (10 men, 27 women, $73.0 \%$ women) who responded to the advertisement for participation in the present study were selected as the intervention group. We include 42 subjects as controls (11 men, 31 women, $73.8 \%$ women) who were matched to the subjects in the intervention group.

In the exercise group, 2 participants had a history of depression, 3 were dyslipidemia, and 3 had the metabolic syndrome. In the control group, 2 participants had a history of depression, 3 were dyslipidemia and 2 had the metabolic syndrome. The data were analyzed by the Chi-square test in order to confirm that the two groups were well matched and

\section{Table 1. Baseline Characteristics of Participants}

\begin{tabular}{lccc}
\hline Variables & Exercise group & Control group & $\mathrm{p}$ \\
\hline $\mathrm{n}$ & 31 & 36 & \\
Men/Women & $8 / 23$ & $10 / 26$ & $0.539^{\mathrm{a}}$ \\
Age (year) & $45.5 \pm 2.2$ & $45.8 \pm 1.2$ & $0.934^{\mathrm{b}}$ \\
Height $(\mathrm{cm})$ & $161.9 \pm 1.4$ & $160.4 \pm 0.9$ & $0.368^{\mathrm{b}}$ \\
BMI $\left(\mathrm{kg} / \mathrm{m}^{2}\right)$ & $23.5 \pm 0.7$ & $22.4 \pm 0.4$ & $0.189^{\mathrm{b}}$ \\
\% body fat $(\%)$ & $26.0 \pm 1.2$ & $26.6 \pm 1.1$ & $0.708^{\mathrm{b}}$ \\
Waist circumference $(\mathrm{cm})$ & $84.6 \pm 2.0$ & $80.6 \pm 1.3$ & $0.095^{\mathrm{b}}$ \\
Weight $(\mathrm{kg})$ & $62.1 \pm 2.2$ & $58.5 \pm 1.2$ & $0.497^{\mathrm{b}}$ \\
\hline
\end{tabular}

Values are expressed as means $\pm \mathrm{SE}$, a, using Chi-square test, $b$, using Mann-Whitney U test, BMI, body mass index, body fat percentage, $\%$ body fat. that our hypotheses were tenable $(\mathrm{p}=0.539)$.

\section{Adherence to aerobic exercise}

Of the 37 participants in the exercise group, 31 (83.8\%) completed the 12-week program (8 men, 23 women, mean age $45.5 \pm 2.2$ years, $74.2 \%$ women, mean BMI $23.5 \pm 0.7 \mathrm{~kg}$ / $\mathrm{m}^{2}$ ), while 36 in the control group $(85.7 \%)$ were available for follow-up at the end of the 12-week study period (10 men, 26 women, mean age $45.8 \pm 1.2$ years; $72.2 \%$ women, mean BMI $22.4 \pm 0.4 \mathrm{~kg} / \mathrm{m}^{2}$ ) (Table 1). The attendance rate for the aerobic exercise program ranged between $43 \%$ to $84 \%$ for each period.

\section{Effect of exercise training on outcome variable}

In the exercise group, weight, \% body fat, waist circumference, WHO-QOL 26 score, triglyceride, total cholesterol, HDL cholesterol and LDL cholesterol had improved significantly by the end of the third month (Table 2). Of the 12 variables measured in the aerobic exercise group, (5 body composite variables, WHO-QOL 26 score, and 6 blood test variables), 4 variables improved significantly (Fig. 1). The aerobic exercise caused significant improvements in weight (men; F=3.766, p=0.0152, women; $\mathrm{F}=3.834$, $\mathrm{p}=0.0083$ ), waist circumference (men; $\mathrm{F}=4.400, \mathrm{p}=0.0075$, women; $\mathrm{F}=$ 2.830, $\mathrm{p}=0.0335$ ), WHO-QOL 26 score (men; $\mathrm{F}=7.080, \mathrm{p}=$ 0.0005 , women; $\mathrm{F}=6.120, \mathrm{p}=0.0004)$, and HDL cholesterol (men; $\mathrm{F}=5.840, \mathrm{p}=0.0017$, women; $\mathrm{F}=3.732, \mathrm{p}=0.0095$ ).

\section{Correlation with HMW adiponectin}

As shown in Table 3, there was a significant negative correlation between HMW adiponectin and body composition (BMI; $\mathrm{r}=-0.509, \mathrm{p}=0.037)$, LDL cholesterol $(\mathrm{r}=-0.578, \mathrm{p}=$ $0.015)$, and fasting plasma glucose $(r=-0.559, p=0.020)$. In

Table 2. The Effects of Aerobic Exercise on the Exercise Group and Control Group during 3 Months

\begin{tabular}{|c|c|c|c|c|c|c|c|}
\hline & \multicolumn{4}{|c|}{ Exercise group $(\mathrm{n}=31)$} & \multicolumn{3}{|c|}{ Control group $(n=36)$} \\
\hline & Pre & 3 months later & $\mathrm{p}$ & & Pre & 3 months later & $\mathrm{p}$ \\
\hline Weight (kg) & $62.1 \pm 2.2$ & $60.8 \pm 2.1$ & 0.001 & $*$ & $58.5 \pm 1.2$ & $57.5 \pm 1.1$ & 0.381 \\
\hline $\operatorname{BMI}\left(\mathrm{kg} / \mathrm{m}^{2}\right)$ & $23.5 \pm 0.7$ & $23.2 \pm 0.7$ & 0.249 & & $22.4 \pm 0.4$ & $22.3 \pm 0.5$ & 0.825 \\
\hline$\%$ body fat $(\%)$ & $26.0 \pm 1.2$ & $25.1 \pm 1.2$ & 0.008 & $*$ & $31.3 \pm 0.7$ & $29.5 \pm 1.0$ & 0.169 \\
\hline Waist circumfercnce $(\mathrm{cm})$ & $84.6 \pm 2.0$ & $80.1 \pm 1.8$ & 0.001 & $* *$ & $80.6 \pm 1.3$ & $81.1 \pm 2.0$ & 0.183 \\
\hline Left holding power (kg) & $30.6 \pm 1.9$ & $31.3 \pm 2.0$ & 0.329 & & $30.8 \pm 1.7$ & $31.0 \pm 1.9$ & 0.945 \\
\hline Right holding power (kg) & $33.2 \pm 1.9$ & $33.5 \pm 1.9$ & 0.455 & & $31.9 \pm 1.7$ & $32.1 \pm 1.8$ & 0.455 \\
\hline WHO-QOL 26 score & $2.9 \pm 0.1$ & $3.1 \pm 0.1$ & 0.001 & $* *$ & $2.9 \pm 0.1$ & $2.9 \pm 0.1$ & 0.878 \\
\hline Triglyceride (mg/dL) & $135.3 \pm 14.5$ & $5121.5 \pm 19.4$ & 0.019 & $*$ & $110.1 \pm 8.7$ & $107.7 \pm 8.7$ & 0.523 \\
\hline Total cholesterol (mg/dL) & $210.8 \pm 6.3$ & $194.7 \pm 5.5$ & 0.002 & $* *$ & $217.1 \pm 5.8$ & $217.4 \pm 5.9$ & 0.600 \\
\hline HDL cholesterol (mg/dL) & $61.0 \pm 3.0$ & $71.8 \pm 2.9$ & 0.001 & $* *$ & $70.0 \pm 2.2$ & $72.3 \pm 2.4$ & 0.080 \\
\hline LDL cholesterol (mg/dL) & $119.7 \pm 5.7$ & $112.9 \pm 4.9$ & 0.037 & $*$ & $122.1 \pm 5.1$ & $120.8 \pm 5.0$ & 0.768 \\
\hline Fasting plasma glucose $(\mathrm{mg} / \mathrm{mL})$ & $97.1 \pm 4.5$ & $95.2 \pm 2.4$ & 0.931 & & $96.9 \pm 3.7$ & $102.9 \pm 3.7$ & 0.073 \\
\hline
\end{tabular}

Values are means \pm SE. $* \mathrm{p}<0.05 * * \mathrm{p}<0.005$, using Wilcoxon signed-rank test. BMI, body mass index, body fat percentage, $\%$ body fat. 

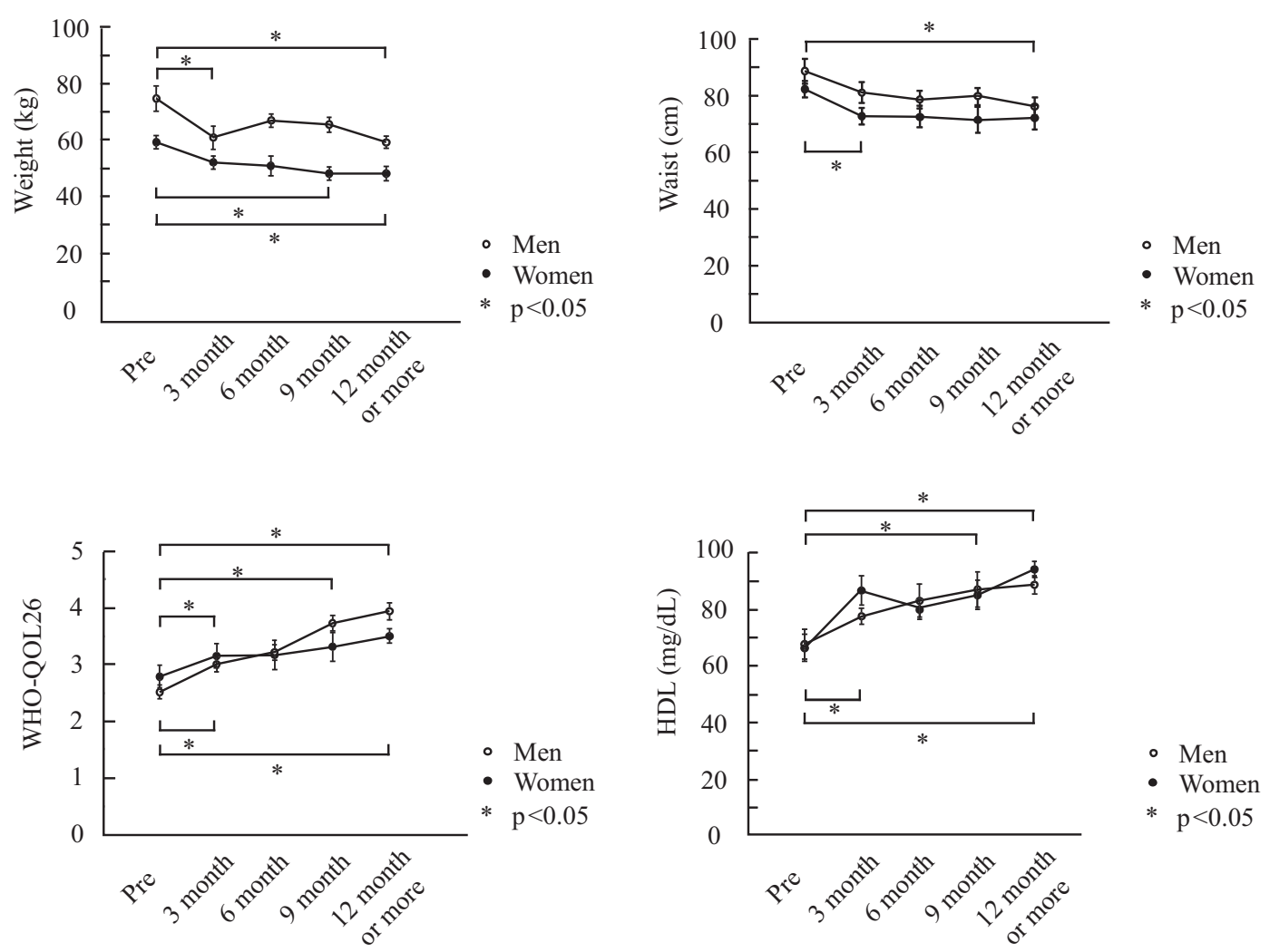

Figure 1. Changes in body composition and blood with the exercise group. Values are means \pm SE. *p $<0.05$, using two-way repeated measures analysis of variance (ANOVA). Numbers of men were as follows: pre, $(n=8) 3$ months, $(n=8), 6$ months, $(n=8), 9$ months, $(n=7), 12$ months or more, $(n=5)$. Numbers of women: pre, $(n=23), 3$ months, $(n=23), 6$ months, $(n=20), 9$ months, $(n=17), 12$ months or more, $(\mathbf{n}=11)$.

Table 3. Pearson's Correlation Analysis and Multiple Regression Analysis of HMW Adiponectin and Other Factors

\begin{tabular}{|c|c|c|c|c|c|}
\hline & \multicolumn{3}{|c|}{$\begin{array}{l}\text { Pearson's correlation analysis } \\
\qquad(\mathrm{n}=17)\end{array}$} & \multicolumn{2}{|c|}{$\begin{array}{l}\text { Multiple regression analysis } \\
\qquad(\mathrm{n}=17)\end{array}$} \\
\hline & Pearson's Y & $\mathrm{p}$ & & $\beta$-values & $\mathrm{p}$ \\
\hline BMI & -0.509 & 0.037 & * & -0.174 & $0.018 *$ \\
\hline$\%$ body fat & -0.031 & 0.907 & & -0.088 & 0.453 \\
\hline Waist circumference & -0.180 & 0.488 & & 0.101 & 0.244 \\
\hline WHO-QOL26 & 0.207 & 0.426 & & 0.040 & 0.213 \\
\hline Triglyceride & -0.093 & 0.722 & & 0.024 & 0.361 \\
\hline Total cholesterol & -0.309 & 0.228 & & 0.014 & 0.114 \\
\hline HDL cholesterol & 0.665 & 0.004 & $* *$ & 0.389 & $0.002 * *$ \\
\hline LDL cholesterol & -0.578 & 0.015 & $*$ & -0.443 & $0.008 *$ \\
\hline Fasting plasma glucose & -0.559 & 0.020 & * & -0.306 & $0.010 *$ \\
\hline $\mathrm{R}^{2}$ & & & & & 0.761 \\
\hline Adjusted $\mathrm{R}^{2}$ & & & & & 0.454 \\
\hline
\end{tabular}

Values are means $\pm \mathrm{SE} . * \mathrm{p}<0.05, * * \mathrm{p}<0.005$, using pearson's correlation analysis and multiple regression analysis. $\mathrm{R}^{2}=$ coefficient of determination. $\mathrm{BMI}$, body mass index, body fat percentage, $\%$ body fat.

contrast, there was a significantly positive correlation between HMW adiponectin and HDL cholesterol $(\mathrm{r}=0.665$, $\mathrm{p}=$ 0.004). There was no relationship between HMW adiponectin and WHO-QOL 26 score, triglyceride, total cholesterol levels, $\%$ body fat, and waist.
Multiple regression analysis examined the relationship between HMW adiponectin and the other factors. There was a significant correlation between HMW adiponectin and body composition (BMI; $\beta=-0.174, \mathrm{p}=0.018$ ), HDL cholesterol ( $\beta=0.389, p=0.002)$, LDL cholesterol $(\beta=-0.443, p=0.008)$, 


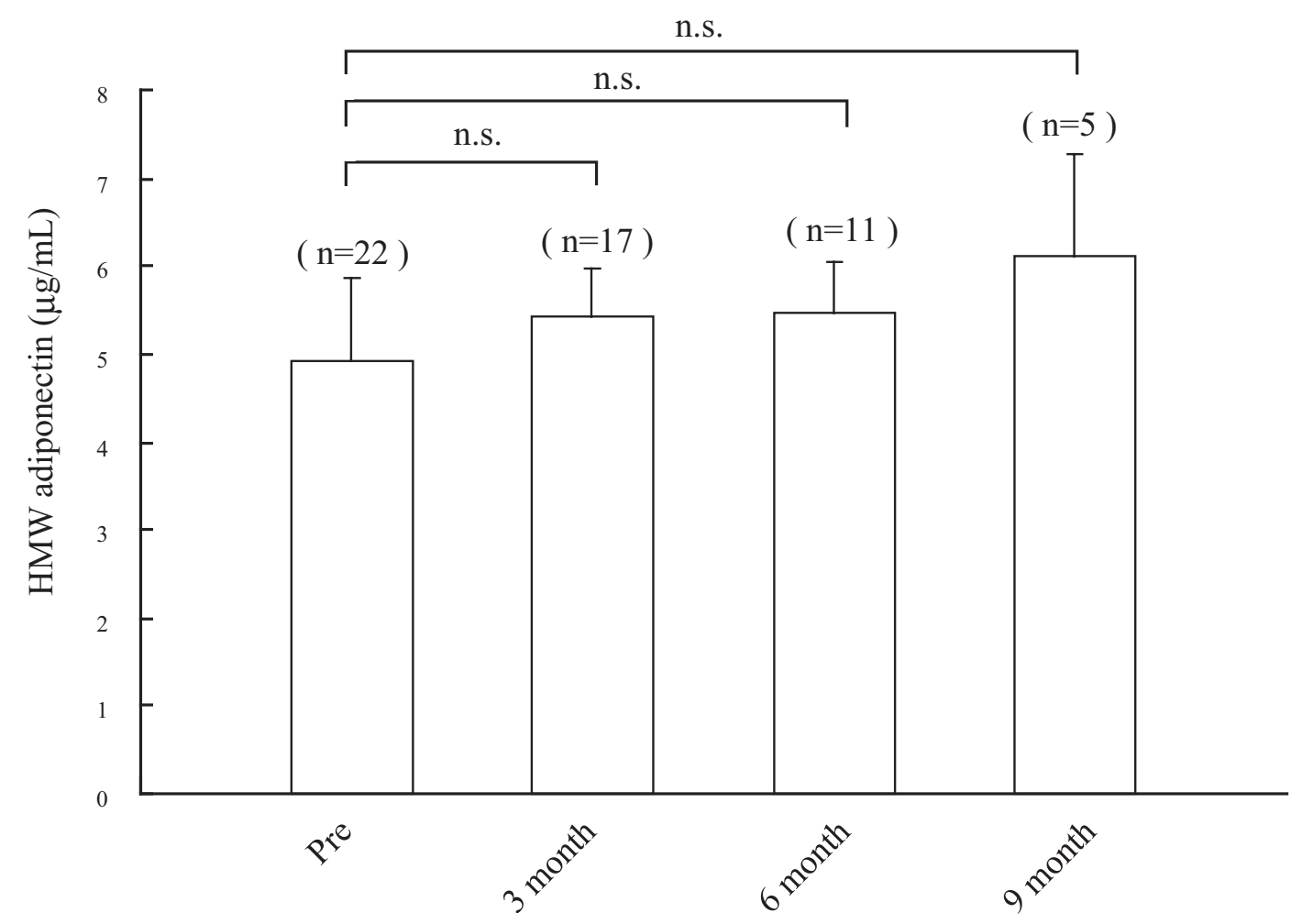

Figure 2. Changes in HMW adiponectin responses with the exercise group during the exercise and post exercise. Values are means \pm SE. n.s., $p>0.05$, using the Mann-Whitney $U$ test. Numbers of participants were as follows: pre, $(n=22), 3$ months, $(n=17), 6$ months, $(n=11), 9$ months, $(n=5)$.

and fasting plasma glucose $(\beta=-0.306, p=0.010$, Coefficient of determination $\mathrm{R}^{2}=0.761$, and adjusted $\mathrm{R}^{2}=0.454$ ). There was still no relationship between HMW adiponectin and WHO-QOL 26 score, triglyceride, total cholesterol levels, \% body fat, and waist.

\section{Adiponectin responses}

The concentration of HMW adiponectin increased in the exercise group, although the difference between pre- and post-exercise levels did not reach statistical significance (Fig. 2). The HMW adiponectin concentration was increased by $24 \%$ after 9 months of exercise compared with preexercise levels.

\section{Discussion}

Aerobic exercise improved body weight, waist circumference, WHO-QOL 26 score, and HDL cholesterol. The concentration of HMW adiponectin increased during the aerobic exercise program, with a significant negative correlation being observed between HMW adiponectin and body composition (BMI). HMW adiponectin also showed a significant positive correlation with HDL cholesterol and a significant negative correlation with LDL cholesterol. We found that the $31(83.8 \%)$ participants who completed the exercise program had significant improvements in weight, \% body fat, waist circumference, WHO-QOL 26 score, triglyceride, total cholesterol, HDL cholesterol and LDL cholesterol at the end of the third month. These findings are in agreement with a previous study on endurance exercise training that reported positive changes in lipid and lipoprotein metabolism (16).

It is generally considered that aerobic exercising has several beneficial effects on health. For example, regular exercise may promote chronic positive effects such as an improvement in lipid profiles, insulin resistance, BMI, and \% body fat, as well as basal metabolic state (17). As the metabolism of lipoproteins occurs mainly during the aerobic exercise (18), the positive changes in anthropometric variables observed in the present study indicate that periodical aerobic exercising has the potential to improve the lipid profile. The pathological changes in the lipid and glucose metabolism in metabolic syndrome are closely related to the state of insulin resistance, unfortunately we have no data of fasting insulin concentration (IRI); further studies are needed to rectify the relation between IRI and exercise.

A loss of body mass and fat has also been associated with decreases in total and LDL cholesterol and an increase in HDL cholesterol. The improvement in total cholesterol may result from the exchange of cholesterol esters between tissues and lipoproteins to HDL cholesterol. Furthermore, exercise training usually decreases the concentration of fasting plasma triglycerides. As a consequence, the levels of very low density lipoprotein (VLDL) decrease and HDL cholesterol levels tend to increase (19). It is considered beneficial to increase the clearance of VLDL and triglyceride, in order to decrease the mean residence time of these lipoproteins in 
the circulation. The concentration of LDL cholesterol is regulated by the balance between synthesis in the liver and removal from the plasma by lipoprotein receptors, while HDL concentration is determined by both HDL cholesterol and apolipoprotein A-I (apoA-I) concentrations. HDL cholesterol concentration increases frequently in response to a decrease in triglyceride, although in this study exercise training did not change the triglyceride levels. Therefore, another possible mechanism for the exercise-induced rise in HDL cholesterol rise we observed may be induction of apoA-I synthesis. There is further evidence that lecithin and cholesterol acyltransferase (LCAT) activity are changed by exercise training, and may therefore also affect HDL cholesterol levels before and after exercise training (20-22).

In this study we showed that continued exercise training led to further improvements in weight, waist circumference, WHO-QOL 26 score and HDL cholesterol levels (Fig. 1) while the other variables remained stable. We also demonstrated that HMW adiponectin correlated positively with HDL cholesterol, and negatively with LDL cholesterol and fasting plasma glucose. It has been reported that HMW adiponectin is not only the more active form of the adipokine $(23,24)$, but is also related closely to insulin sensitivity in the metabolic syndrome $(25,26)$. The present study investigated the role of aerobic exercise and improvement in lipid profiles in relationship to changes in HMW adiponectin concentration. Blüher et al (27) found that HMW adiponectin concentration increased with exercise treatment even in subjects with normal glucose tolerance. In contrast, Bobbert et al (28) showed that HMW adiponectin concentration was similar before and after 6 weeks of marathon training without a reduction in body weight. In the present study, HMW adiponectin concentration increased in the exercise group, although this increase did not reach significance during the aerobic exercise program (Fig. 2). The effect of aerobic exercise on HMW adiponectin concentration therefore remains to be established conclusively. However, HMW adiponectin concentrations tended to be higher than in the pre-exercise period. Further studies are needed to determine whether HMW adiponectin concentration changes significantly during longer periods of aerobic exercise or with different intensity of exercises.

\section{Conclusion}

Aerobic exercise leads to an improvement in body composition and lipid profile. The concentration of HMW adiponectin also showed a tendency to improve with regular aerobic exercise compared with pre-exercise levels.

The authors state that they have no Conflict of Interest (COI).

\section{Acknowledgement}

We would like to thank the Saga Shimbun Culture Center.

\section{References}

1. Phillips GB. Sex hormones, risk factors and cardiovascular disease. Am J Med 65: 7-11, 1978.

2. Reaven GM. Role of insulin resistance in human disease. Diabetes 37: 1595-1607, 1988.

3. Grundy SM. Obesity, metabolic syndrome and cardiovascular disease. J Clin Endocrinol Metab 89: 2595-2600, 2004.

4. Kawada $\mathrm{T}$, Otsuka $\mathrm{T}$, Inagaki $\mathrm{H}$, et al. Increase in the prevalence of metabolic syndrome among workers according to age. Aging Male 13: 184-187, 2010.

5. Ford ES, Giles WH, Dietz WH. Prevalence of the metabolic syndrome among US adults: findings from the third National Health and Nutrition Examination survey. JAMA 287: 356-359, 2002.

6. Das UN. Metabolic syndrome X: an inflammatory condition? Curr Hypertens Rep 6: 66-73, 2004.

7. Haffner SM. The metabolic syndrome: inflammation, diabetes mellitus and cardiovascular disease. Am J Cardiol 97: 3A-11A, 2006.

8. Leon AS, Connett J, Jacobs DR Jr, Rauramaa R. Leisure-time physical activity levels and risk of risk of coronary heart disease and death: the Multiple Risk Factor Intervention Trail. JAMA 258: 2388-2395, 1987.

9. Pate RR, Pratt M, Blair SN, et al. Physical activity and public health. A recommendation from the Centers for Disease Control and Prevention and the American College of Sports Medicine. JAMA 273: 402-407, 1995.

10. Eaton CB, Lapane KL, Garber CE, Assaf AR, Lasater TM, Carleton RA. Physical activity, physical fitness, and coronary heart disease risk factors. Med Sci Sports Exerc 27: 340-346, 1995.

11. Hsieh SD, Yoshinaga H, Muto T, Sakurai Y. Regular physical activity and coronary risk factors in Japanese men. Circulation 97: 661-665, 1998.

12. Iso H, Sato S, Kitamura A, et al. Metabolic syndrome and the risk of ischemic heart disease and stroke among Japanese men and women. Stroke 38: 1744-1751, 2007.

13. Karvonen MJ, Kentala E, Mustala O. The effects of training on heart rate; a longitudinal study. Ann Med Exp Biol Fenn 35: 307$315,1957$.

14. WHO-QOL GROUP. Development of WHO QOL: Rational and current status. Int J Ment Health 23: 24-56, 1994.

15. Nakano Y, Tajima S, Yoshimi A, et al. A novel enzyme-linked immunosorbent assay specific for high-molecular-weight adiponectin. J Lipid Res 47: 1572-1582, 2006.

16. Durstine JL, Grandjean PW, Davis PG, Ferguson MA, Alderson NL, DuBose KD. Blood lipid and lipoprotein adaptations to exercise: a quantitative analysis. Sports Med 31: 1033-1062, 2001.

17. Sharma AM. Effects of exercise on plasma lipoproteins. N Engl J Med 348: 1494-1496, 2003.

18. Kelley GA, Kelley KS. Effects of aerobic exercise on non-highdensity lipoprotein cholesterol in children and adolescents: a metaanalysis of randomized controlled trials. Prog Cardiovasc Nurs 23: 128-132, 2008.

19. Ring-Dimitriou S, von Duvillard SP, Paulweber B, et al. Nine months aerobic fitness induced changes on blood lipids and lipoproteins in untrained subjects versus controls. Eur J Appl Physiol 99: 291-299, 2007.

20. Khabazian BM, Ghanbari-Niaki A, Safarzadeh-Golpordesari A, Ebrahimi M, Rahbarizadeh F, Abednazari H. Endurance training enhances ABCA1 expression in rat small intestine. Eur J Appl Physiol 107: 351-358, 2009.

21. Ghanbari-Niaki A, Khabazian BM, Hossaini-Kakhak SA, Rahbarizadeh F, Hedayati M. Treadmill exercise enhances ABCA1 expression in rat liver. Biochem Biophys Res Commun 361: 841-846, 2007.

22. Olchawa B, Kingwell BA, Hoang A, et al. Physical fitness and re- 
verse cholesterol transport. Arterioscler Thromb Vasc Biol 24: 1087-1091, 2004.

23. Waki H, Yamauchi T, Kamon J, et al. Impaired multimerization of human adiponectin mutants associated with diabetes. Molecular structure and multimer formation of adiponectin. J Biol Chem 278: 40352-40363, 2003.

24. Hada Y, Yamauchi T, Waki H, et al. Selective purification and characterization of adiponectin multimer species from human plasma. Biochem Biophys Res Commun 356: 487-493, 2007.

25. Hara K, Horikoshi M, Yamauchi T, et al. Measurement of the high-molecular weight form of adiponectin in plasma is useful for the prediction of insulin resistance and metabolic syndrome. Diabetes Care 29: 1357-1362, 2006.

26. Katsuki A, Suematsu M, Gabazza EC, et al. Decreased high- molecular weight adiponectin-to-total adiponectin ratio in sera is associated with insulin resistance in Japanese metabolically obese, normal-weight men with normal glucose tolerance. Diabetes Care 29: 2327-2328, 2006.

27. Blüher M, Brennan AM, Kelesidis $T$, et al. Total and highmolecular weight adiponectin in relation to metabolic variables at baseline and in response to an exercise treatment program: comparative evaluation of three assays. Diabetes Care 30: 280-285, 2007.

28. Bobbert T, Wegewitz U, Brechtel L, et al. Adiponectin oligomers in human serum during acute and chronic exercise: relation to lipid metabolism and insulin sensitivity. Int J Sports Med 28: 1-8, 2007.

(C) 2011 The Japanese Society of Internal Medicine http://www.naika.or.jp/imindex.html 